\section{Ingeniería}

CONTENIDO

Artículos

Análisis numérico de un sistema de aire acondicionado empleando
mallas estructuradas y no estructurades A

Funciones de regresión para caudales extremos en la Vertiente Rojas, Nazareth; Aguilar, José Francisco; Solís, Hernán. Evaluación de daños por agresión ambiental en viviendas
de concreto reforzado. Solís, Rómel; Moreno, Eric; Jiménez, Felipe; Rosas, Victorino.

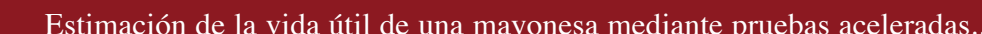
Estimación de la vida útil de una mayonesa
García, Claudia; Molina, Manuel.

Inestabilidad oscilatoria de tensión debido a los motores de inducción …….....................65-75 rde, Gustavo; Araya, Eddie.

Estabilidad y colapso de tensión en sistemas eléctricos Araya, Eddie.

Obtención de pulpa a la soda antraquinona de Tectona grandis creciendo en Costa Rica...........................

Protocolo evaluador para el manejo de desechos y la seguridad ocupacional
aplicado al laboratorio clínico del Hospital San Francisco de Asís de Grecia. Ruiz, Francisco.

9. Euler: su contex
Vargas, Celso.

10. Reserva de resistencia de edificios porticados de concreto armado disenaados conforme al ACI-318/ IBC 2006 ........ Análisis y comentarios

Herrera, Rodolfo.

breve semblanza.

Trabajos de Graduación 2007

Lista de proyectos de graduación de grado y posgrado

Normas

$\sum_{\text {EDTORAL }}$

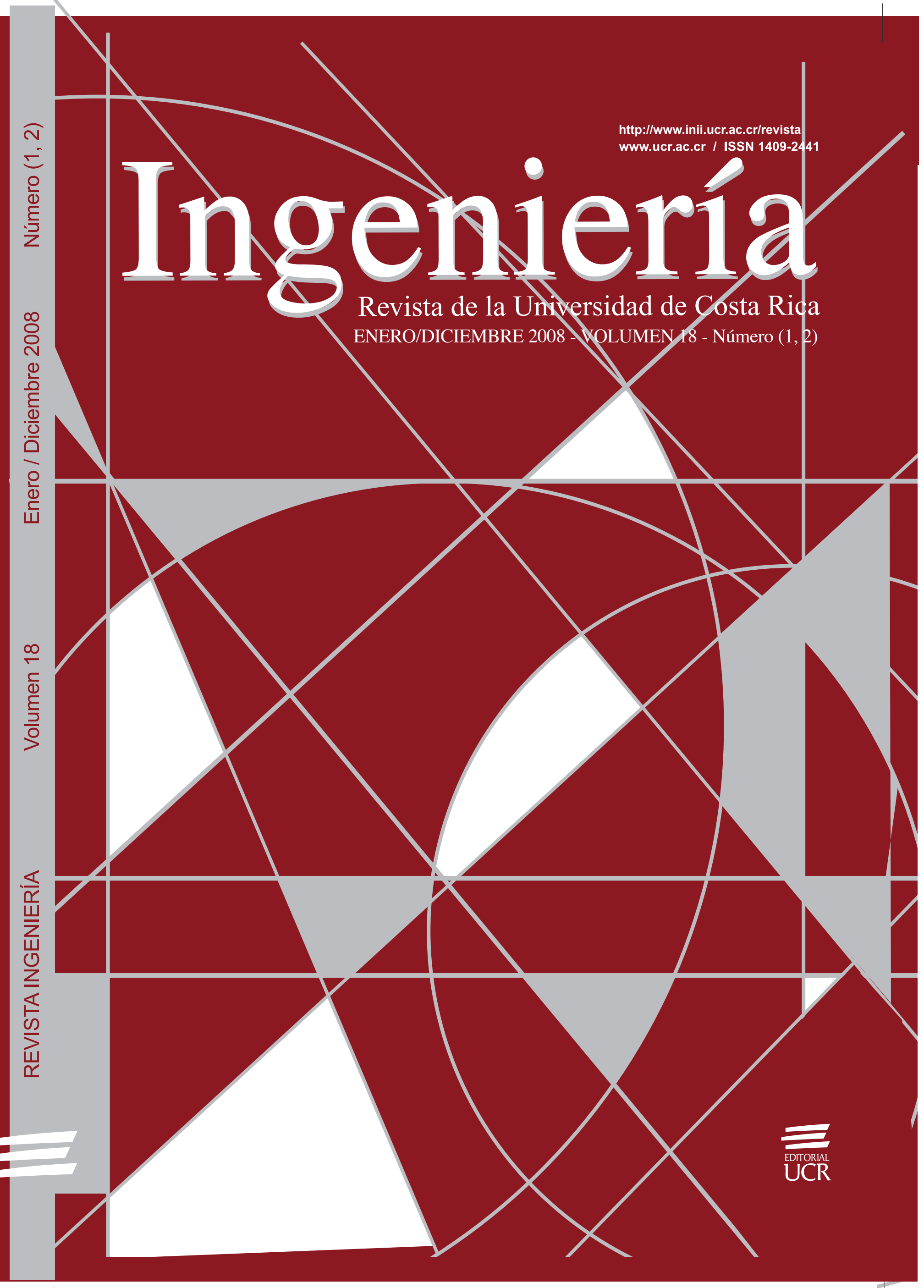




\section{Análisis y comentarios}





\title{
LUIS GONZÁLEZ GONZÁLEZ: UNA BREVE SEMBLANZA ${ }^{1}$
}

\author{
Rodolfo Herrera Jiménez
}

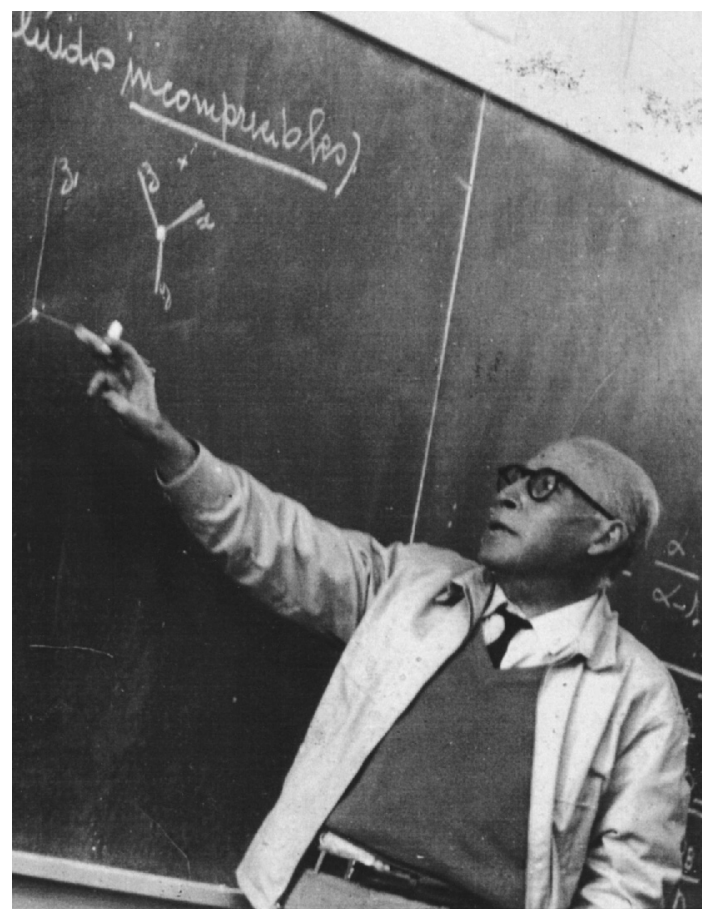

Figura 1. Luis González González dictando clases, década del sesenta.

Fuente: (Foto cedida por el Ph. D. Rodolfo Herrera Jiménez).
Hoy solamente me ocuparé, brevemente, de resaltar algunos aspectos sobre la figura de Luis González González. que, a mi criterio, son relevantes y necesarios para poder situarlo históricamente en la cultura universitaria actual.

Pienso que posiblemente a don Luis le hubiera gustado vivir en la isla Laputa, descrita por Swift en el tercer viaje de Gulliver, la cual se sostenía magnéticamente en la atmósfera, acompañada de esa especie de raza que la habitaba totalmente dedicada "a las matemáticas y a la música", con "...uno de sus ojos vuelto al interior y el otro mirando directamente al cenit".

Aunque don Luis era sordo y no hubiera podido escuchar notas concretas, posiblemente "oiría" "notas abstractas", producidas por una especie de "intuición intelectual", como él mismo afirmaba. En sus palabras: "el matemático puede "ver" esos espacios con solo mirar sus ecuaciones, en una forma semejante a como el músico puede "oír" una sinfonía con sólo mirar la partitura".

Él pensaba igual que lo escrito por Galileo en su famoso párrafo del Saggiatore: "La ciencia natural está escrita en este gran libro, que se encuentra siempre abierto ante nuestros ojos - me refiero al universo - pero no lo podréis entender hasta que no lleguéis a comprender su lenguaje y hasta que no conozcáis los símbolos en que está escrito. Está escrito en lenguaje matemático, y los símbolos son triángulos, círculos, y otras figuras geométricas, sin las cuales sería imposible para el hombre aprender su contenido".

Si bien no es monopolio de la actividad científica ni la inteligencia, ni la curiosidad, ni el razonamiento, ni la paciencia, ni el esfuerzo, pues igualmente se pueden encontrar estas cualidades en alguien dedicado a cualquier otra actividad, sí lo es el fundamento y el método matemático, un rasgo característico que presentan las ciencias de Occidente, en especial la ciencia que cultivó don Luis, como la mecánica racional o como la llama C. Truesdell, la filosofía natural moderna.

Muchas veces se me ha preguntado ¿cuál fue la actividad principal a la que se dedicó don Luis?, ¿fue filósofo, matemático, ingeniero o científico?. Estas clasificaciones son un problema de nuestro tiempo en el que se trata, por la deformante especialización, de encasillar a ciertas personalidades como la que nos ocupa, en algún recipiente cerrado. En primera instancia, debo decir que fue un humanista y un insigne 
un insigne maestro, más cercano a un hombre de la Ilustración. A pesar que fue ingeniero de profesión, graduado en la École de Ponts et Chauseé de Bruxelles, Bélgica, como ingeniero trabajó muy poco, pues se dedicó la mayor parte de su vida a la enseñanza y a la exposición e investigación en el campo de las ciencias. Sin embargo, en la Facultad de Ingeniería fue siempre el consultor gratuito de ingenieros y profesores, quienes requerían de su saber universal en distintos campos de las ciencias de la ingeniería.

Por otra parte, algunos no lo consideran matemático ya que no siguió estudios formales en tal disciplina, a mi criterio un punto de vista erróneo, no muy acorde con la historia de la ciencia y que es propio de considerar a la matemática como ciencia pura e independiente de las ciencias. Aparte de que en ese tiempo los estudios que un ingeniero seguía en las escuelas de ingeniería latinas europeas, eran iguales a los matemáticos y análogamente con los cursos de mecánica racional, etc., el nivel de exigencia era muy alto. En todo caso, a lo largo de su vida intelectual amplió su conocimiento en esta ciencia.

Otros reclamarán que no fue un físico, por las mismas razones anteriores o porque no se dedicó a lo que algunos creen, que la física consiste solo en el estudio de las mal llamadas "partículas elementales" o porque simplemente nunca trabajó en un laboratorio. Sin embargo, basta leer su libro sobre turbinas o los textos de mecánica racional que escribió, incluyendo un tomo en mecánica de fluidos, para darse cuenta del alto conocimiento que tenía de esta ciencia.

En realidad don Luis, aparte de la enseñanza, en la que fue un verdadero maestro, se dedicó al estudio de la mecánica racional y sus aplicaciones necesarias para las ciencias de la ingeniería, como las distintas ramas aplicadas de lo que hoy Clifford Ambrose. Lubliner denomina la termomecánica de los cuerpos deformables. Para él, la mecánica racional era una ciencia matemática, lo cual no significaba que fuera un campo de las matemáticas, en sus aspectos puramente formales. Entendía muy bien la diferencia entre la matemática pura, como ciencia formal sin referentes ni interpretación fáctica, y la matemática como instrumento constitutivo del pensar, como el lenguaje de la ciencia fáctica, como un elemento indispensable en la elaboración racional, en la construcción cognoscitiva de la verdad.

En este campo de la ciencia la palabra "matemáticas" no ha de entenderse en el sentido de matemáticas "puras", divorciadas de la naturaleza, sino como la actividad intelectual que asigna magnitudes matemáticas, generalmente números, figuras geométricas, u operaciones delimitadas por reglas formales, a fenómenos naturales y al posterior uso del más estricto razonamiento matemático para reemplazar las cualidades, tendencias, medidas experimentales y reglas empíricas típicas de las ciencias de otras culturas, así como las actividades artesanales que aparecen en toda cultura.

Lo que no es más que el método y el camino iniciado por Newton y Euler, en el campo de las ciencias naturales y exactas. La verdad es que las teorías modernas de la ciencia de la mecánica han sido desarrolladas por escuelas en ciencias de la ingeniería o de matemáticas, al igual que en el tiempo de la Ilustración; la mecánica clásica como afirma Clifford Ambrose. Truesdell no fue descubierta por los físicos, sino por un puñado de geómetras o algebristas, quienes, al igual que representaban a una esfera material con una esfera matemática, también representaban un tipo de cuerpo material y sus leyes internas con un modelo matemático. Construir una filosofía natural matemática en la cual la geometría es tan solo la parte más sencilla, si bien una muestra perfecta del método.

No participó de la ansiosa credulidad de aquellos profanos que insisten en considerar a la ciencia como algo mágico, revelada por inspiración mística de unos pocos elegidos. Si la verdadera escuela del método científico es el estudio de los maestros, él tuvo como tales a grandes geómetras, cristianos o agnósticos: Descartes, Pascal, Newton, Leibnitz, Bernoullis, Euler, Lagrange, Comte, Einstein y también de todos quienes estudian, enseñan o admiran la prodigiosa ciencia de las grandezas, de las formas, de los números, de las medidas, de las funciones, de los movimientos y de las fuerzas.

Su intención de escribir sobre tensores de la manera que creía más didáctica, le nació por su interés en la teoría de la relatividad, interés que lo transmitió a algunos de sus discípulos. Él consideraba que la mayoría de las exposiciones sobre la llamada teoría relativista de Einstein entraba en dificultades apenas aparecían los tensores, pues la forma de generar estos conceptos no era la más conveniente. En 
sobre la teoría de la relatividad coincide con la interpretación del astrónomo francés Nordman (1922) quien escribió al respecto: "Mientras que para entender a Spinoza no es necesario sino saber un poco de latín, monstruos espantosos montan guardia ante Einstein y se esfuerzan con horribles muecas en impedir el acceso. Se agitan detrás de móviles y extrañas rejas, tan pronto rectangulares, tan pronto curvilíneas, que se llaman coordenadas. Llevan nombres tan monstruosos como ellos mismos. Llámense vectores contravariantes y covariantes, tensores, escalarios, determinantes, vectores ortogonales, símbolos de tres índices generalizados, etc. ¡qué se yo!. Todos estos seres, importados del fondo más salvaje de la selva matemática, se agrupan o se subdividen con una extraña promiscuidad por esas asombrosas cirugías que se llaman la integración y la diferenciación. En una palabra, si Einstein es un tesoro, la horrible legión de reptiles matemáticos aleja de él al curioso". Tal es en parte, la razón de don Luis por su interés didáctico respecto a los tensores, expresado en múltiples trabajos y lecciones. Mi libro, publicado en su honor, denominado Vectores, Afinores y Tensores, es una expresión tardía de parte de sus investigaciones y su metodología matemática. Libro publicado por la Editorial de la Universidad de Costa Rica, con el apoyo del ex-decano de la Facultad de Ciencias, el ingeniero y matemático don Manuel A Calvo H., uno de sus ex-alumnos.

Estudió la teoría de la relatividad einsteniana, sin la cual, decía, no se puede entender el mundo moderno. En relación con ella hizo un trabajo filosófico, denominado Meditaciones en torno a la relatividad publicado en la Revista de Filosofía de esta Universidad, en el que explica cómo algunos de los conceptos del cambio paradigmático que produjo Einstein, son en realidad el producto del análisis epistemológico, de "una profunda meditación filosófica" realizada sobre los conceptos de espacio y tiempo. También, en otros trabajos mostró la profundidad filosófica de su pensamiento, como por ejemplo el denominado La axiomática moderna y el principio de identidad, articulo también publicado en la Revista Filosofía de esta Universidad. Estos trabajos fueron analizados por el suscrito en el Congreso de Filosofía realizado en esta Universidad en 1984.

Fue el primer profesor de cálculo infinitesimal y de mecánica racional que tuvo el país, y como curiosidad académica se puede anotar que fue el primero que aplicó la "variable compleja" a problemas de geodesia, en un trabajo hecho para el Instituto Geográfico Nacional en 1938 y publicado por quien les habla en la Revista Ingeniería. Fue el primer profesor de tiempo completo de la Facultad y en realidad para servicio de la Facultad de Ciencias y Letras como auxiliar en la enseñanza de los cursos de Humanidades, específicamente los desarrollados por el profesor visitante Dr. Roberto Saumells, relacionados con la revolución cultural que implicó el nacimiento de las geometrías no euclideanas y la teoría de la relatividad. Simultáneamente dictaba sus lecciones de Mecánica Racional y Cálculo Infinitesimal en Ingeniería. Su labor durante la época de la llamada revolución cultural producida por la reforma de Rodrigo Facio Brenes nunca ha sido reconocida en los medios culturales del país, posiblemente debido a su callada función y a la concepción de lo que es la cultura.

En la enseñanza de la matemática se enfrentaba al formalismo exagerado que partía de sistemas axiomatizados y que fue moda común por allá de las décadas de los cincuenta a los sesenta. Al respecto decía que la axiomática “... no es un método de investigación, sino simplemente un método de exposición racional de una ciencia ya hecha. Viene a ser una sublimación del método deductivo, en la que este ha sido despojado de sus elementos ontológicos. El método axiomático reduce las matemáticas a un inmenso "herbario" lógicamente clasificado, en el cual puede uno encontrar todo lo que la vida intelectual ha creado; todo, excepto la vida misma. La matemática axiomatizada puede crecer como crecen los herbarios, por adición de nuevos especímenes. Pero no crecerá como las plantas de los jardines, por el hálito de vida que llevan en sí mismas". Sin embargo, no despreciaba el espíritu de la axiomática en matemáticas, pues decía que ha influido favorablemente en su desarrollo.

Respecto a la lógica formal escribía que “... es muy buena, y hasta puede ser útil, mientras no se la tome demasiado en serio. Es decir, mientras no se la considere como algo absoluto e infalible, hasta el punto de que llegue a convertirse en una verdadera atadura para el entendimiento". Concibió con claridad los límites ontológicos de la lógica formal, cuando afirmó que “... el punto vulnerable de 
la lógica es que está basada en hipótesis metafísicas, a saber, que existen leyes a priori de la razón, que estas leyes son absolutas, inmutables y eternas", en contraste con el aspecto evolutivo de todas las nociones humanas, es decir, frente a la dialéctica real del movimiento de las cosas. Análogamente con la matemática, que al referirse a constructos comentaba “... se mudó de casa, abandonó el mundo de la ontología y se fue a vivir al país de la lógica pura, aséptica, impoluta, sin contaminación alguna con el mundo de la realidad sensible". Siendo así, decía que era ampliamente especulativa, pues puede "inventar" sus constructos sin referencia alguna a los objetos concretos.

¿Fue un investigador?. Antes de responder hay que observar que este vocablo, tan utilizado hoy día, ha sido sobredimensionado o tergiversado. Investigar significa descubrir algo desconocido o no bien conocido, es la búsqueda metodológica del conocimiento, la exploración cognoscitiva en algún campo del saber. Investigar científicamente un hecho o problema no es en sí un estado de beatitud. Es una actividad típicamente ausente de la seudo-ciencia y de la ideología.

Bajo el lema de "obligación o servicio social" la "universidad social-comercial" convierte a todo maestro de ciencia en una "máquina de hacer ciencia", olvidándose en primer lugar de la razón de por qué es hecha la investigación. Eso permite un liberalismo de la universidad tal, que cualquier cuestión sea por definición declarada un objeto de investigación académica. El resultado es que se confunda con cualquier búsqueda de datos, bibliografía o un escaneo en la red o con cualquier proceso de enseñanza.

Como se puede interpretar de los comentarios anteriores, la personalidad y el pensamiento de don Luis están muy lejos del mundo de la universidad social-comercial y de la investigación masiva de hoy. Si estuviera entre nosotros seguramente lucharía por no permitir que no se destruya el aprendizaje profundo, y que la investigación con metas puramente utilitarias no elimine nuestro derecho a la capacidad de pensar.

Luis González fue un profesor humilde tratando de hacer ciencia, y la más prodigiosa, en la Costa Rica de las décadas de los treinta a los sesenta, atrasada culturalmente en especial en las matemáticas y más aun en la mecánica racional o en la filosofía natural. En esa época las facilidades para obtener información eran muy pobres, de modo que el aislamiento intelectual era muy grande. Sin embargo con su trabajo tesonero, su actividad de maestro, su conocimiento y su cultura, fue un pionero y pilar para estimular en algunos el amor por la matemática y las ciencias matemáticas, proyectando sus esfuerzos en la creación de la "Carrera de Matemáticas" de esta Universidad. Entre otras cosas políticas apoyó la trágica denuncia de Einstein sobre la Responsabilidad del Intelectual en el mundo moderno, publicando el texto en su artículo para la Revista Filosofía de esta Universidad.

$\mathrm{Su}$ actividad, su oficio de conocer y enseñar, fue una escuela de moral, cumplió siempre con los principios que señala el Dr. Mario Bunge: las virtudes esenciales, su código interno: la honestidad intelectual, el culto a la verdad e independencia de juicio, el coraje intelectual, el amor por la libertad intelectual y el sentido de justicia. Además en su condición de maestro, transmitió a sus discípulos el disfrute del orden, la precisión, la elegancia y la belleza estética y del especial "sabor" que contiene el lenguaje matemático.

Don Luis fue un querido y singular amigo, con quien siempre se podían tratar temas rigurosamente metafísicos y quien nos abrió a muchos el encuentro con proposiciones matemáticas, y el saber percibirlas con la capacidad de asombro ante su dimensión estética. El diálogo sigue a través de mi memoria, pues cuando las preguntas y las respuestas se llevan en el pensamiento y el sentimiento, el diálogo puede continuar.

¿Acaso no nos decía usted, don Luis, que la visión pitagórica fundamental, la base de su sistema, consistió en la persuasión profunda de la inteligibilidad del cosmos mediante el número? tal y como lo escribió en su himno al número, en uno de los pocos fragmentos que han llegado hasta nosotros de uno de los pitagóricos primitivos: Filolao, y que dice: "Grande, todopoderosa, todo perfeccionadora y divina es la fuerza del número, comienzo y regidor de la vida divina y humana, participante en todo. Sin el número todo es confuso y oscuro. Porque la naturaleza del número proporciona conocimiento y es guía 
y maestra para todos en todo lo que es dudoso y desconocido. Porque nada de las cosas nos serían claras si no existiera el número y su esencia. Este es quien armoniza en el alma las cosas con su percepción, haciéndolas cognoscibles y congruentes unas con otras, según su naturaleza, proporcionándoles corporeidad (Diels B.11).

Por medio de esta cátedra, la Facultad de Ingeniería y la Universidad le reconocen en algo lo que su actividad callada y sin el estruendo de la investigación dominante hoy, lo que le entregó a la cultura matemática y a las ciencias exactas en nuestro país, con su exquisita labor como pensador, investigador y maestro de ciencias exactas.

\section{NOTAS}

1 Esta semblanza fue presentada por el autor en la Cátedra Conmemorativa Luis González, en la Lección inaugural del II ciclo lectivo de la Facultad de Ingeniería y la reapertura de la Cátedra, 29 de agosto del 2007.

\section{SOBRE EL AUTOR}

\section{Rodolfo Herrera Jiménez}

Doctor en Ingeniería Civil.

Profesor Emérito de la Universidad de Costa Rica. Miembro del Consejo del Postgrado en "Ciencia Cognoscitiva" de la Univessidad de Costa Rica; miembro del Consejo del Sistema Acreditación de la Educación Superior (SINAES), Costa Rica.

Teléfono: (506) 2253-4549, 2224-7090.

Apartado: 106-2050

Correo electrónico: rodolfoh@racsa.co.cr. 